\title{
Homogenization Models for a Simple Dielectric-Composite Slab upon Oblique Incidence
}

\author{
Jiaran Qi, Jinghui Qiu, and Chongzhi Han \\ Department of Microwave Engineering, School of Electronics and Information Engineering, Harbin Institute of Technology, \\ P.O. Box 3054, No. 2 Yi Kuang Street, Nan Gang District, Harbin 150080, China
}

Correspondence should be addressed to Jiaran Qi; qi.jiaran@hit.edu.cn

Received 2 September 2013; Revised 2 December 2013; Accepted 2 December 2013; Published 2 January 2014

Academic Editor: Tat Yeo

Copyright (C) 2014 Jiaran Qi et al. This is an open access article distributed under the Creative Commons Attribution License, which permits unrestricted use, distribution, and reproduction in any medium, provided the original work is properly cited.

Four different models are applied to effectively describe a geometrically simple dielectric-composite slab. The corresponding model parameters, when the oblique incidence is taken into account, are retrieved based on the transmission and reflection data and compensated with the nonmagnetic assumption. The scattering parameters of each model with derived parameters for various angles of incidence are then analytically calculated using the forward propagation matrix method and compared with the simulated scattering parameters from the real composite slab. According to these comparisons, it is shown that spatial dispersion makes it challenging to achieve angle-independent parameters for the applied four models. Moreover, when a stratified model is employed to describe the composite slab of our interest under oblique incidence, the boundary layers need to be anisotropic.

\section{Introduction}

Homogenization of a given composite whose heterogeneity scale is sufficiently small is a long-lasting problem of continuing interest [1-5]. In this so-called long-wavelength regime, various characterization techniques have been developed to determine the macroscopic constitutive properties of composites, for instance, different mixing formulas [1, $2,6]$, field averaging method [7], and scattering parameter (S-parameter) retrievals [8-11]. It is usually sufficient in this regime to characterize the macroscopic electromagnetic properties of composites using the homogeneous and isotropic model with the effective permittivity $\varepsilon_{\text {eff }}$ and the effective permeability $\mu_{\text {eff }}$.

However, in the study of metamaterials [5], homogenization is more challenging since the size of unit cell or the scale of geometrical detail is often an appreciable fraction of the effective wavelength inside the materials. Recent studies have revealed that, in such a metamaterial regime between the quasistatic one and the photonic crystal one, refractive index is well defined while wave impedance turns out to be less rigorous due to nonlocality or spatial dispersion [12-16]. This point evokes researchers to question the validity of classic homogenization theory in the metamaterial regime [13-16].
It was found, for instance, that, for a fishnet metamaterial, all retrieved parameters strongly depend on the angle of incidence (or lateral wave vector component) [16]. Nevertheless, these efforts concentrated on the case that either the structure under investigation is illuminated normally by a plane wave, or only the homogeneous model is used to approximate the macroscopic electromagnetic properties of the structure.

If a certain homogenization model is applied to describe the macroscopic electromagnetic properties of a composite, it is crucial to examine its validity by evaluating the performance of the model when a plane wave excitation with different incidence angles is considered. Two schemes exist for achieving such a goal. One is to first analytically calculate the $S$-parameters, at oblique incidence, for the model whose parameters are resolved at normal incidence. By comparing at various incidence angles the differences between the above-calculated $S$-parameters and those from the real composite by full wave simulation, one can study whether the model performance varies with altering angle of incidence. The other one is to examine whether the retrieved model parameters depend on the incidence angle, which requires the generalization of the classic retrieval methods derived at normal incidence into oblique incidence. 


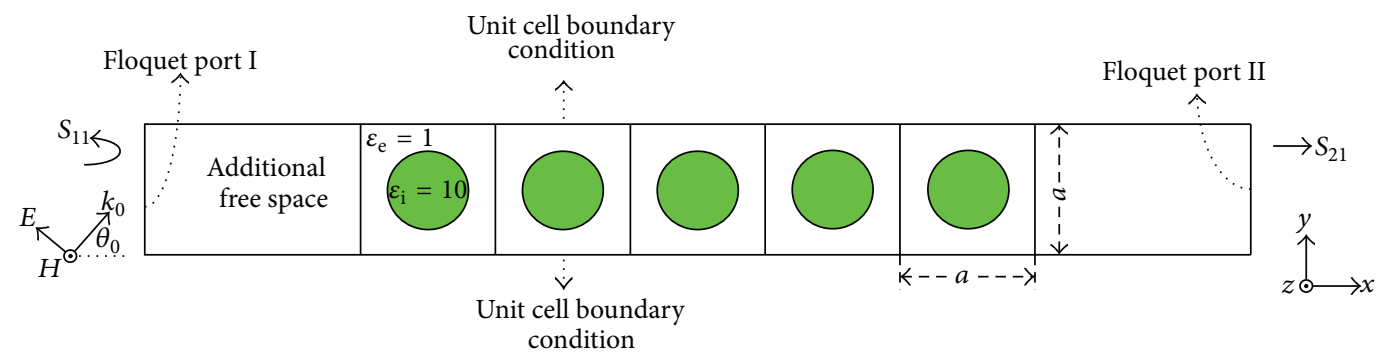

FIgURE 1: The quasi-2D geometry of the dielectric slab constructed in CST MWS, where the slab thickness in the $z$-direction is a/100 to reduce the computational complexity, and the unit cell boundary condition is assigned to the $y$-and $z$-bounds. The environment and inclusion permittivities are denoted as $\varepsilon_{e}$ and $\varepsilon_{i}$, while $\theta_{0}$ is the incidence angle of the $\mathrm{TM}_{x y}$-polarized plane wave.

On the other hand, many reported composites are geometrically asymmetric $[17,18]$. It is also revealed that the outermost boundary layers of a periodic structure with finite thickness display different properties from the inner layers [19-21]. These facts make necessary the attempt to introduce homogenization models more complicated than the classic homogeneous one. Hence, it is necessary to study the performances of different homogenization models by taking into account oblique incidence.

Instead of focusing on a certain metamaterial, we consider in this paper a simple dielectric-composite slab illuminated obliquely by a plane wave. One reason is that a reliable quasistatic estimate for the effective permittivity of such a slab is available as a good reference for our retrieval results. Moreover, such a simple geometry reduces many uncertainties so that fundamental limitations of each applied model could be more clearly revealed. Aside from the classic homogeneous model, three different homogenization models are employed to effectively describe the composite slab, and the dependence of their performance on the angle of incidence is evaluated. It is worth mentioning that all the quasidynamic homogenizations are performed when the effective wavelength inside the slab is at least 10 times the dimension of its unit cell. Within such a region, the $S$ parameter retrieval in [11] is generalized for oblique incidence and anisotropic model using new formulations different from those in [22]. In particular, a compensation method is presented to restore the retrieved parameters that are obscured by the resonances due to the finite thickness of the dielectric slab. Moreover, a 2-layer method and a numerical inversion approach are originally proposed and applied to determine the permittivities of the boundary layers and the inner layers for the stratified homogenization models.

The contents of this paper are arranged as follows. In Section 2, the geometrical setup of the dielectric slab of our interest is illustrated. Also, the Lord Rayleigh estimate for its static permittivity is introduced. In Section 3, the applied four homogenization models and the corresponding retrieval methods for model parameters are presented. Based on the presented methods, characteristic parameters of each model are retrieved at oblique incidence in Section 4. Furthermore, according to the model evaluation procedure introduced in this section, we compare the performance of each homogenization model, in order to demonstrate the advantages of newly proposed models and also to reveal their limitations. Finally, conclusive remarks are given in Section 5.

\section{Geometry Setup and the Lord Rayleigh Prediction}

The dielectric slab of interest, with $z$-directed circular cylindrical inclusions whose relative permittivity $\varepsilon_{i}$ is 10 and volume fraction $p$ is 0.3 , is shown in Figure 1. We hereafter only consider a $\mathrm{TM}_{x y}$-polarized plane wave with an incidence angle $\theta_{0}$ as the incoming electromagnetic (EM) field. The slab in this two-dimensional (2D) problem is composed of five layers of square unit cells in the $x$-direction with side length $a$ and relative permittivity $\varepsilon_{e}(=1)$ and forms an infinite lattice in the $y$-direction. We construct this slab in the full wave simulator CST Microwave Studio (MWS) where the unit cell boundary condition is allocated to the four bounds in the $y$ - and $z$-directions. By varying the phase shifts between the unit cell boundary pairs in the $y$-direction and selecting the proper mode, a $\mathrm{TM}_{x y}$-polarized plane wave with a specific angle of incidence can be realized. Moreover, additional free space is added to prevent the higher modes from propagating and the Floquet ports are used to terminate the structure in the $x$-direction, generate the excitation, and collect the desired $S$-parameters.

For an infinite lattice corresponding to the slab in Figure 1, the analytical 2D Lord Rayleigh formula can accurately predict the static effective permittivity $\varepsilon_{\text {Ray }}$ of the lattice [23]:

$$
\begin{aligned}
\varepsilon_{\mathrm{Ray}}=\varepsilon_{e}+\left(2 p \varepsilon_{e} \times(\right. & \left(\varepsilon_{i}+\varepsilon_{e}\right)\left(\varepsilon_{i}-\varepsilon_{e}\right)^{-1}-p \\
& -\left(0.3058 \cdot p^{4}+0.0134 \cdot p^{8}\right) \\
& \left.\left.\times\left(\varepsilon_{i}-\varepsilon_{e}\right)\left(\varepsilon_{i}+\varepsilon_{e}\right)^{-1}\right)^{-1}\right) .
\end{aligned}
$$

Although not exactly accurate for the finite-thickness slab, the Lord Rayleigh formula still provides a fairly good validation for our retrieved permittivities. 


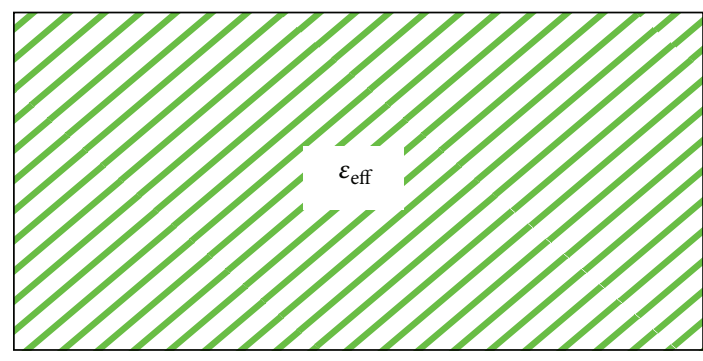

(a) The homogeneous isotropic model (H-model)

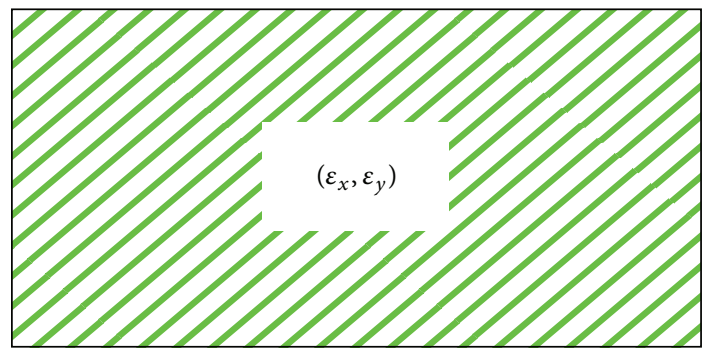

(c) The anisotropic model (A-model)

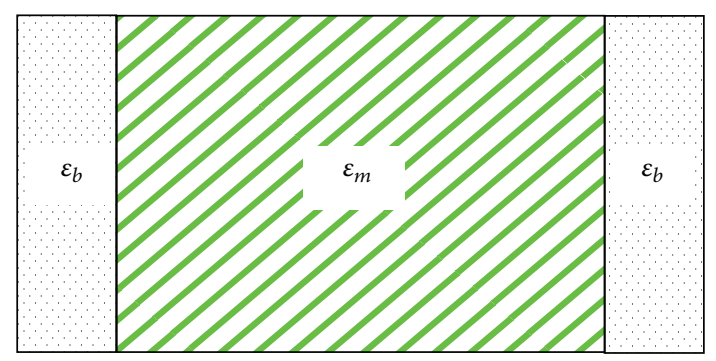

(b) The stratified model with isotropic boundary layers (IBLmodel)

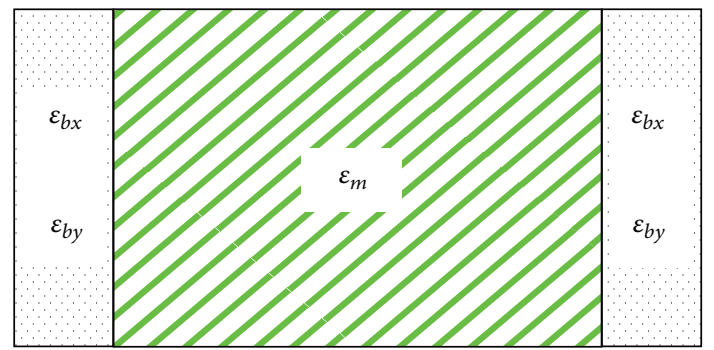

(d) The stratified model with anisotropic boundary layers (ABL-model)

FIGURE 2: Four different models applied to characterize the dielectric slab in Figure 1. In (a), $\varepsilon_{\text {eff }}$ is the relative effective permittivity of the homogeneous model; $\varepsilon_{b}$ and $\varepsilon_{m}$ in (b) are the permittivities of the outermost boundary and inner layers; $\varepsilon_{x}$ and $\varepsilon_{y}$ in (c) denote the $x$-and $y$-components of the permittivity; $\varepsilon_{b x}$ and $\varepsilon_{b y}$ in (d) are the $x$-and $y$-components of the permittivities of the outermost boundary layers, while $\varepsilon_{m}$ is the inner layer permittivity.

Moreover, we normalize the frequency to a reference $f_{20}$, which denotes the frequency when the effective wavelength $\lambda_{\text {eff }}$ in the slab is 20 times the length of the unit cell. We define $\lambda_{\text {eff }}$ by reducing the free space wavelength $\lambda_{0}$ according to the static Lord Rayleigh prediction, that is, $\lambda_{\text {eff }}=\lambda_{0} /\left(\varepsilon_{\text {Ray }}\right)^{1 / 2}$. The normalized frequency $f / f_{20}$ then not only displays the frequency dependence of the retrieved model parameters but also roughly indicates the ratio between the unit cell size and the effective wavelength.

\section{Parameter Retrieval Methods for Different Models}

The most straightforward model for the slab shown in Figure 1 is a homogeneous isotropic medium ( $\mathrm{H}$-model) with effective permittivity $\varepsilon_{\text {eff }}$ in Figure 2(a). According to our recent studies [21] and previous literature [19, 20, 24], the permittivity of the outermost boundary layer (with only one neighbor in the $x$-direction) is, however, different from those of the inner layers (with neighbors on both sides). A physically sounder stratified model with isotropic boundary layers (IBL-model) is therefore employed and shown in Figure 2(b). On the other hand, when oblique incidence is considered, the isotropic models may no longer characterize the finite-thickness slab sufficiently. Hence, two anisotropic models in Figures 2(c) and 2(d) are introduced, namely, the anisotropic model (A model) and the stratified model with anisotropic boundary layers (ABL model). In particular, we only make the boundary layers anisotropic to keep the overall model reasonably simple in Figure 2(d), as suggested in [20].

3.1. Generalized Nicolson-Ross-Weir (NRW) Method for an Anisotropic Slab at Oblique Incidence. When a plane wave is normally incident on a homogeneous slab, the medium parameters can be obtained based on the reflection and transmission data. This classic approach is commonly known as the Nicolson-Ross-Weir (NRW) method [8,9], which has been further improved in $[10,11]$. In particular, our recent study [25] shows that for a homogeneous lossless dielectric composite the compensation method is required besides the NRW method to acquire the reasonable permittivities at normal incidence. We will in this paper give the generalized NRW and compensation methods for the situation when a $\mathrm{TM}_{x y}$-polarized plane wave is obliquely incident on an anisotropic slab.

In this case, the $S$-parameters can be formulated as follows:

$$
\begin{gathered}
S_{11}=\frac{R\left(1-e^{-j 2 k_{1} d \cos \theta_{1}}\right)}{1-R^{2} e^{-j 2 k_{1} d \cos \theta_{1}}}, \quad S_{21}=\frac{\left(1-R^{2}\right) e^{-j k_{1} d \cos \theta_{1}}}{1-R^{2} e^{-j 2 k_{1} d \cos \theta_{1}}}, \\
R=\frac{k_{1} \cos \theta_{1}-\varepsilon_{y} k_{0} \cos \theta_{0}}{k_{1} \cos \theta_{1}+\varepsilon_{y} k_{0} \cos \theta_{0}},
\end{gathered}
$$

where $k_{0}$ and $k_{1}$ are the wave numbers in the vacuum and the slab, while $\theta_{0}$ and $\theta_{1}$ are the corresponding incidence angles. 
The $y$-component of the permittivity and the thickness of the slab in the $x$-direction are denoted as $\varepsilon_{y}$ and $d$. If we introduce the generalized impedance $z^{\prime}$ and refractive index $n^{\prime}$ as follows:

$$
\begin{gathered}
z^{\prime}=\frac{k_{1} \cos \theta_{1}}{\varepsilon_{y} k_{0} \cos \theta_{0}}= \pm \sqrt{\frac{\left(1+S_{11}\right)^{2}-S_{21}^{2}}{\left(1-S_{11}\right)^{2}-S_{21}^{2}}} \\
n^{\prime}=\frac{k_{1}}{k_{0}} \cos \theta_{1}=\frac{1}{k_{0} d}\{[-\operatorname{Im}[\ln (Q)]+2 q \pi]+j \operatorname{Re}[\ln (\mathrm{Q})]\} \\
Q=\frac{S_{21}}{1-S_{11}\left(\left(z^{\prime}-1\right) /\left(z^{\prime}+1\right)\right)}
\end{gathered}
$$

where the integer $q$ denotes the branch of the logarithmic function, $z^{\prime}$ and $n^{\prime}$ will then be related to the $S$-parameters through (3)-(5). The medium parameters can thus be calculated.

3.1.1. Homogeneous Model. For a homogenous slab in Figure 2(a) illuminated obliquely by a plane wave, the permittivity $\varepsilon_{\text {eff }}$ and permeability $\mu_{\text {eff }}$ read according to (3) and (4)

$$
\varepsilon_{\mathrm{eff}}=\frac{n^{\prime}}{\left(z^{\prime} \cos \theta_{0}\right)}, \quad \mu_{\mathrm{eff}}=\frac{z^{\prime} n^{\prime} \cos \theta_{0}}{\cos ^{2} \theta_{1}}
$$

Also from Snell's law, we have

$$
\sin ^{2} \theta_{1}=\frac{\sin ^{2} \theta_{0}}{\left(\varepsilon_{\mathrm{eff}} \mu_{\mathrm{eff}}\right)}
$$

Equations (6) and (7) are sufficient to determine $\varepsilon_{\text {eff }}$ and $\mu_{\text {eff }}$, which are, however, distorted by the Fabry-Pérot resonances (FPRs) $[25,26]$. The FPR, present when the slab width is an integer multiple of half of the effective wavelength in the slab, is actually introduced through $z^{\prime}$ and has no influence on $n^{\prime}$. This fact indicates that the influence of the FPRs will be removed if we can retrieve the $\varepsilon_{\text {eff }}$ using only $n^{\prime}$. By substituting (7) into (6), we get

$$
\varepsilon_{\text {eff }}=\frac{\left(n^{\prime 2}+\sin ^{2} \theta_{0}\right)}{\mu_{\text {eff }}} .
$$

Together with the nonmagnetic assumption $\mu_{\mathrm{eff}}=1$, the compensated permittivity of the homogeneous model upon oblique incidence can be obtained.

3.1.2. Anisotropic Model. For an anisotropic medium in Figure 2(c), the dispersion equation in the medium is

$$
\frac{k_{1}^{2} \cos ^{2} \theta_{1}}{\varepsilon_{y}}+\frac{k_{1}^{2} \sin ^{2} \theta_{1}}{\varepsilon_{x}}=\frac{\omega^{2}}{c^{2}},
$$

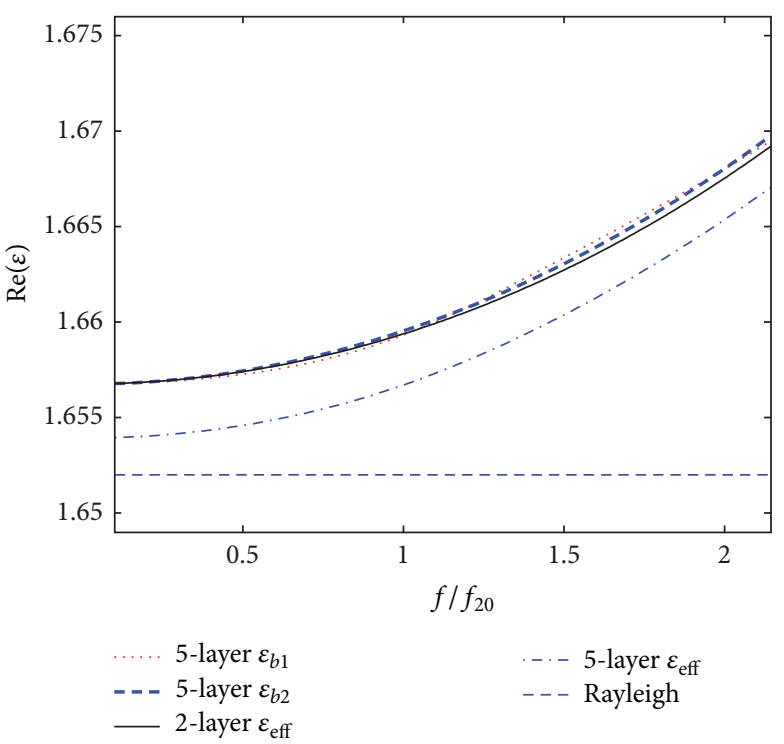

FIgURE 3: The outermost boundary layer permittivities $\varepsilon_{b 1}$ and $\varepsilon_{b 2}$ of a 5-layer slab versus $\varepsilon_{\text {eff }}$ of the $\mathrm{H}$-model for the 2-layer and 5layer slabs. The field averaging method [21] is applied to compute these curves. As defined in Section 2, $f_{20}$ is the frequency when the effective wavelength $\lambda_{\text {eff }}$ in the slab is 20 times the length of the unit cell $a$. The quantity $f / f_{20}$ then not only represents frequency, but also roughly indicates the ratio between $\lambda_{\text {eff }}$ and $a$. For instance, if $f / f_{20}=2$, then $\lambda_{\text {eff }} \approx 10 a$.

where $\varepsilon_{x}$ and $\varepsilon_{y}$ are the $x$ - and $y$-components of the permittivity, and $c$ is the light speed in vacuum. Together with (3) and (4), we have

$$
\begin{gathered}
n^{\prime}=\sqrt{\frac{\varepsilon_{x} \varepsilon_{y}}{\varepsilon_{x} \cos ^{2} \theta_{1}+\varepsilon_{y} \sin ^{2} \theta_{1}}} \cos \theta_{1}, \\
z^{\prime}=\sqrt{\frac{\varepsilon_{x} \varepsilon_{y}}{\varepsilon_{x} \cos ^{2} \theta_{1}+\varepsilon_{y} \sin ^{2} \theta_{1}}} \frac{\cos \theta_{1}}{\varepsilon_{y} \cos \theta_{0}} .
\end{gathered}
$$

Also the phase matching condition gives

$$
\sin ^{2} \theta_{1}=\frac{\varepsilon_{x} \sin ^{2} \theta_{0}}{\varepsilon_{x} \varepsilon_{y}-\left(\varepsilon_{y}-\varepsilon_{x}\right) \sin ^{2} \theta_{0}} .
$$

Finally, we have

$$
\varepsilon_{y}=\frac{n^{\prime}}{\left(z^{\prime} \cos \theta_{0}\right)}, \quad \varepsilon_{x}=\frac{\sin ^{2} \theta_{0}}{\left(1-n^{\prime} z^{\prime} \cos \theta_{0}\right)}=\frac{\varepsilon_{y} \sin ^{2} \theta_{0}}{\left(\varepsilon_{y}-n^{\prime 2}\right)} .
$$

3.2. Retrieval Methods for the Stratified Medium. Several observations have been made in our recent study based on the field averaging method [21] for a dielectric slab shown in Figure 1. Firstly, the permittivities of the outermost boundary layers $\varepsilon_{b 1}$ and $\varepsilon_{b 2}$ are roughly identical. Secondly, all the inner layers have the same permittivity $\varepsilon_{m}$. Thirdly, $\varepsilon_{b 1}$ and $\varepsilon_{b 2}$ are larger than $\varepsilon_{m}$. Finally, for two slabs with different numbers of layers, the permittivities of their outermost boundary layers 
are approximately the same. Figure 3 confirms that $\varepsilon_{b 1}$ and $\varepsilon_{b 2}$ of a 5-layer slab have good agreement with the effective bulk permittivity $\varepsilon_{\text {eff }}$ for a 2-layer slab, where both layers behave like a boundary layer with only one neighbor in the $x$-direction. It is true that quantitatively small differences exist among $\varepsilon_{b 1}, \varepsilon_{b 2}$, and 2-layer $\varepsilon_{\text {eff }}$, but compared with their deviations from the 5-layer $\varepsilon_{\text {eff }}$, these differences can roughly be neglected. We can thus resolve $\varepsilon_{b}$ of the IBL-model in Figure 2(b) or $\varepsilon_{b x}$ and $\varepsilon_{b y}$ of the ABL-model in Figure 2(d) by calculating $\varepsilon_{\text {eff }}$ of the $\mathrm{H}$-model or $\varepsilon_{x}$ and $\varepsilon_{y}$ of the A-model for a 2-layer slab, namely, the 2-layer method.

Once $\varepsilon_{b}$ is known, the inner layer permittivity $\varepsilon_{m}$ can be numerically solved by inverting the formulation of the forward propagation matrix method (FPMM) [27]. For a stratified slab with $t$ layers, there are $t+1$ boundaries which separate the space into $t+2$ regions. Assuming that each region is anisotropic with $\varepsilon_{i, x}$ and $\varepsilon_{i, y}(i=0,1, \ldots, t+1)$, the FPMM then gives the following equation, which reads

$$
\begin{aligned}
& {\left[\begin{array}{c}
S_{21} \cdot \exp \left(j k_{0} d \cos \theta_{0}\right) \\
0
\end{array}\right]} \\
& \quad=D_{(t+1) t} \cdot D_{t(t-1)} \cdots D_{(i+1) i} \cdots D_{10} \cdot\left[\begin{array}{c}
1 \\
S_{11}
\end{array}\right],
\end{aligned}
$$

where $k_{0}$ and $\theta_{0}$ are the wave number and the incidence angle of the incoming plane wave in region 0 (free space) and $d$ is the total thickness of the stratified slab in the $x$-direction. Also, the forward propagation matrix $D_{(i+1) i}$ reads

$$
D_{(i+1) i}=\frac{1+p_{(i+1) i}}{2}\left[\begin{array}{cc}
\exp \left[j d_{i}\left(k_{i+1} \cos \theta_{i+1}-k_{i} \cos \theta_{i}\right)\right] & R_{(i+1) i} \exp \left[j d_{i}\left(k_{i+1} \cos \theta_{i+1}+k_{i} \cos \theta_{i}\right)\right] \\
R_{(i+1) i} \exp \left[-j d_{i}\left(k_{i+1} \cos \theta_{i+1}+k_{i} \cos \theta_{i}\right)\right] & \exp \left[-j d_{i}\left(k_{i+1} \cos \theta_{i+1}-k_{i} \cos \theta_{i}\right)\right]
\end{array}\right]
$$

with

$$
\begin{gathered}
R_{(i+1) i}=\frac{p_{(i+1) i}-1}{p_{(i+1) i}+1}, \quad p_{(i+1) i}=\frac{k_{i} \cos \theta_{i} \cdot \varepsilon_{i+1, y}}{k_{i+1} \cos \theta_{i+1} \cdot \varepsilon_{i, y}}, \\
\sin ^{2} \theta_{i+1}=\frac{\varepsilon_{i+1, x} \sin ^{2} \theta_{i}}{\varepsilon_{i+1, x} \cdot \varepsilon_{i+1, y}-\left(\varepsilon_{i+1, y}-\varepsilon_{i+1, x}\right) \cdot \sin ^{2} \theta_{i}}, \\
k_{i}=k_{0} \sqrt{\frac{\varepsilon_{i, x} \cdot \varepsilon_{i, y}}{\varepsilon_{i, x} \cos ^{2} \theta_{i}+\varepsilon_{i, y} \sin ^{2} \theta_{i}}},
\end{gathered}
$$

where $k_{i}$ and $\theta_{i}$ defined in (16) are the wave number and the propagation angle of the wave in region $i ; R_{(i+1) i}$, caused by the boundary separating the regions $i$ and $i+1$, represents the reflection coefficient for the waves in region $i$; and $d_{i}$ denotes the location of the $i$ th boundary in the $x$-direction. In particular, we assume that $d_{0}=0$.

For the 3-layer slab shown in Figures 2(b) and 2(d) whose boundary layer permittivity is determined, (13) then reduces to a system of two equations with only one unknown the inner layer permittivity $\varepsilon_{m}$, which reads

$$
f_{1}\left(\varepsilon_{m}\right)=S_{11}, \quad f_{2}\left(\varepsilon_{m}\right)=S_{21} .
$$

This enables us to numerically determine the frequency dependence of $\varepsilon_{m}$ by letting the following function reach its minimum at different frequencies,

$$
\begin{aligned}
& \left|\operatorname{Re}\left(f_{1}\left(\varepsilon_{m}\right)-S_{11}\right)\right|+\left|\operatorname{Im}\left(f_{1}\left(\varepsilon_{m}\right)-S_{11}\right)\right| \\
& \quad+\left|\operatorname{Re}\left(f_{2}\left(\varepsilon_{m}\right)-S_{21}\right)\right|+\left|\operatorname{Im}\left(f_{2}\left(\varepsilon_{m}\right)-S_{21}\right)\right| .
\end{aligned}
$$

\section{Results and Discussion}

In order to facilitate understanding, the whole structure of Section 4 is summarized as a flow chart in Figure 4. For two isotropic models, that is, the $\mathrm{H}$ - and IBL-models, the unknown model parameters are first retrieved at normal incidence. The $S$-parameters at different oblique incidence are then calculated by the FPMM for these two models with retrieved parameters at normal incidence and compared with the $S$-parameters from the real composite slab simulated by CST MWS. This step, including the $S$-parameters computation for a derived homogenization model and the comparison with the simulated $S$-parameters from a real structure, is termed as the model evaluation procedure (MEP). If the difference between the calculated $S$-parameter by FPMM and those from simulation is roughly independent of the incidence angle, the model turns out to be sufficient to describe the macroscopic electromagnetic behaviors of the given composite slab. Otherwise, angle-dependent model parameters will be computed.

On the other hand, several attempts are made to find angle-independent parameters for two anisotropic models, that is, the A- and ABL-models. If such efforts fail, the angledependent parameters of these models will be calculated using the methods discussed in Section 3.2. The final step is to make comparisons among three models with angledependent parameters (highlighted by the blue boxes in Figure 4) using MEP to discuss the necessity of the additional complexities, that is, the anisotropy and the separated boundary layer, introduced to the homogenous and isotropic model (H-model).

4.1. Homogeneous Model (H-Model). The permittivity $\varepsilon_{\text {eff }}\left(\theta_{0}=0^{\circ}\right)$ of the $\mathrm{H}$-model at the normal incidence is first retrieved by (8) and shown in Figure 5 as the purple dot-dashed curve. Assuming that the model parameters are independent of the angel of incidence, the $S$-parameters when a $\mathrm{TM}_{x y}$-polarized plane wave is obliquely incident on this $\mathrm{H}$-model with $\varepsilon_{\text {eff }}\left(\theta_{0}=0^{\circ}\right)$ at $0^{\circ}, 30^{\circ}$, and $60^{\circ}$ can be calculated using the FPMM and compared with the simulation results from the real composite slab. For convenience, we define the $S$-parameter difference as 


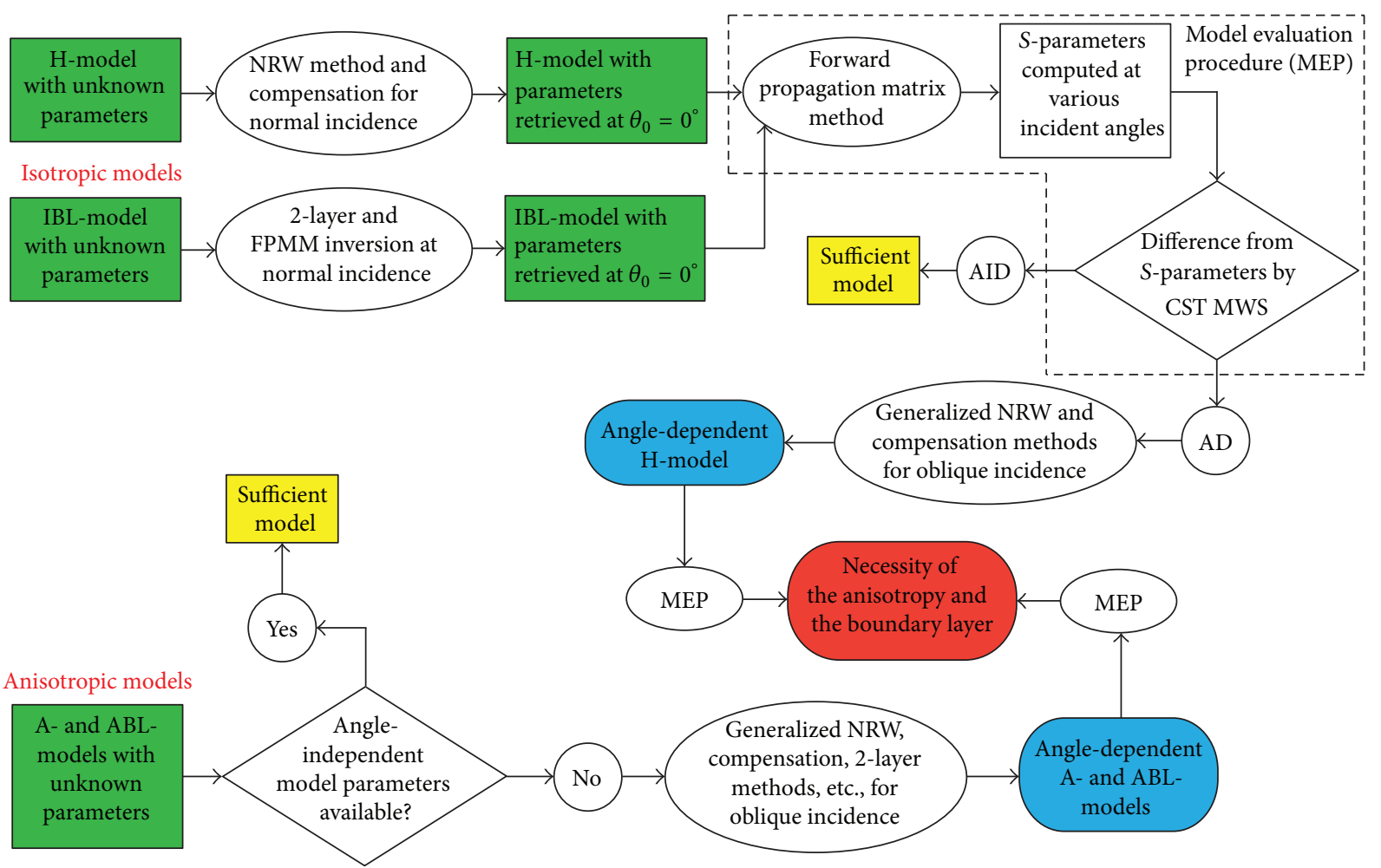

Figure 4: Flow chart as the skeleton of Section 4 where AD and AID stand for angle dependent and angle independent. The process in the dashed box is termed as model evaluation procedure (MEP), including the $S$-parameters computation for a derived model and the comparison with the simulated $S$-parameters from a real composite slab. The reason that the IBL-model is absent from the final comparison highlighted by the red box will be explained in Section 4.5. One can refer the model abbreviations to the definitions in Figure 2.

the sum of the amplitudes of the $S_{11}$ difference and the $S_{21}$ difference; that is, $\left|\Delta S_{11}\right|+\left|\Delta S_{21}\right|$. Such differences, when $\theta_{0}$ respectively, equals $0^{\circ}, 30^{\circ}$, and $60^{\circ}$, are visualized with the red-dashed curves in Figures 6, 7, and 8. It is clear that the performance of this $\mathrm{H}$-model with $\varepsilon_{\text {eff }}$ retrieved at normal incidence deteriorates with increasing incidence angle, which indicates the angle dependence of the parameters of the H-model.

Therefore, the frequency-dependent $\varepsilon_{\text {eff }}$ is calculated for different incidence angles $\theta_{0}$ based on (8) and supplemented into Figure 5. At low frequency, all the curves converge to the same value slightly larger than the Rayleigh prediction (1.6520) and gradually deviate from one another with increasing frequency, which more clearly visualizes the influence due to spatial dispersion. In particular, $\varepsilon_{\text {eff }}$ decreases with increasing $\theta_{0}$ at the same frequency point; for instance, $\varepsilon_{\text {eff }}$ at $\theta_{0}=60^{\circ}$ has the least deviation from the Rayleigh predicted value.

4.2. Stratified Model with Isotropic Boundary Layers (IBLModel). Let us consider the more complicated stratified model with separated isotropic boundary layers. As discussed in Section 3.2, $\varepsilon_{b}$ is first determined by computing $\varepsilon_{\text {eff }}$ of the $\mathrm{H}$-model for a 2-layer slab by (8) at normal incidence. The inner layer permittivity $\varepsilon_{m}$ is then numerically determined according to (13)-(18). It can be seen from Figure 9 that at $\theta_{0}=0^{\circ}$, the inner layer permittivity $\varepsilon_{m, 0}$ (black solid curve)

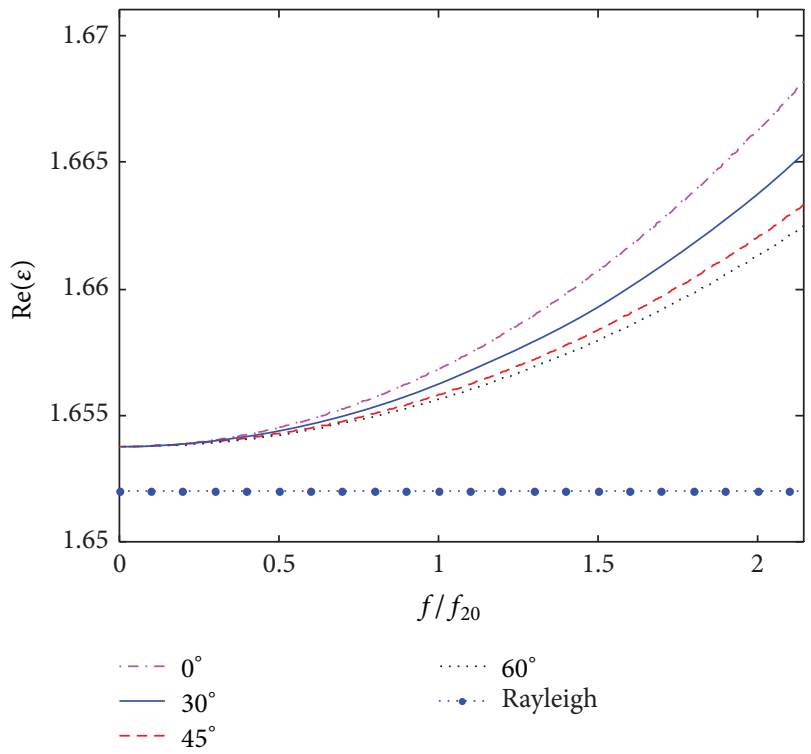

FIGURE 5: The retrieved permittivities as a function of $f / f_{20}$ at different incidence angles, compared with Rayleigh static prediction.

converges to the Rayleigh estimate at low frequencies, while the boundary layers exhibit a larger electric response $\varepsilon_{b, 0}$ (red solid curve). It is also noted that $\varepsilon_{\text {eff } 5 \text { layer }}$ (blue solid curve) of 


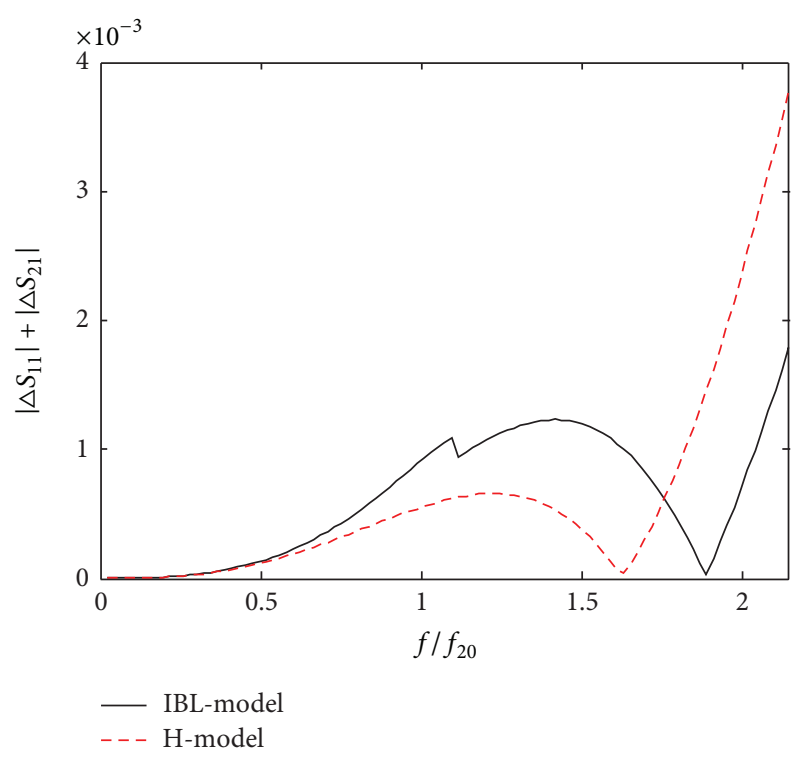

Figure 6: The $S$-parameter differences at $\theta_{0}=0^{\circ}$ for the $\mathrm{H}$ - and IBL-models with parameters retrieved at normal incidence.

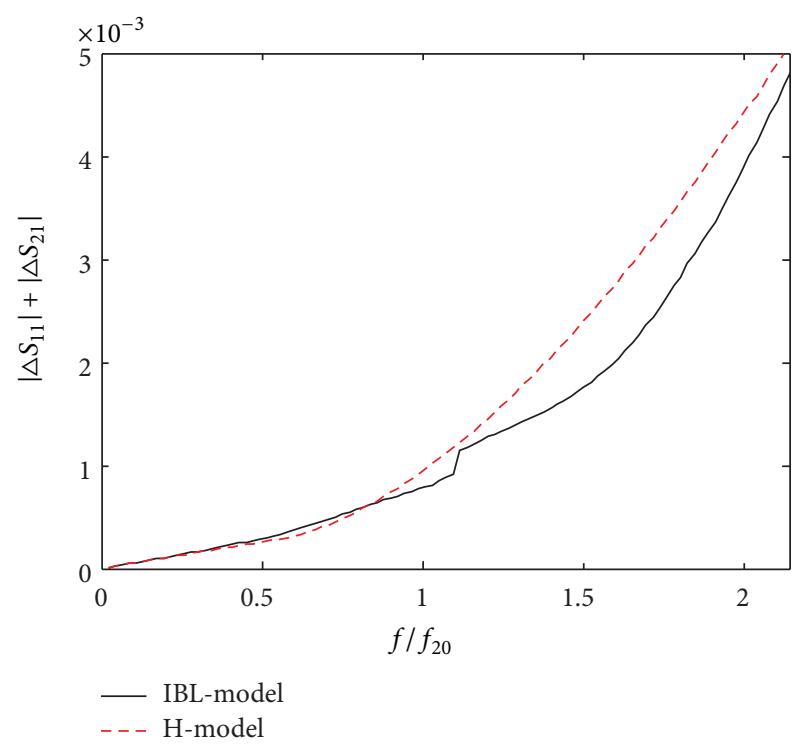

Figure 7: The $S$-parameter differences at $\theta_{0}=30^{\circ}$ for the $\mathrm{H}$ - and IBL-models with parameters retrieved at normal incidence.

the $\mathrm{H}$-model for the 5-layer slab, retrieved by (8) at $\theta_{0}=0^{\circ}$, lies between $\varepsilon_{m, 0}$ and $\varepsilon_{b, 0}$, which confirms the results by field averaging method in [21].

Similar to Section 4.1, the $S$-parameter comparisons are performed when $\theta_{0}=0^{\circ}, 30^{\circ}$, and $60^{\circ}$, and the S-parameter differences $\left|\Delta S_{11}\right|+\left|\Delta S_{21}\right|$ are added into Figures 6-8 as the black solid curves. The IBL-model with parameters retrieved at normal incidence unfortunately fails to display any superiority over the $\mathrm{H}$-model at an arbitrary $\theta_{0}$. Special attention should be paid to the fact that, even for the normal incidence, these two models exhibit the same-level performance although the IBL-model seems physically more

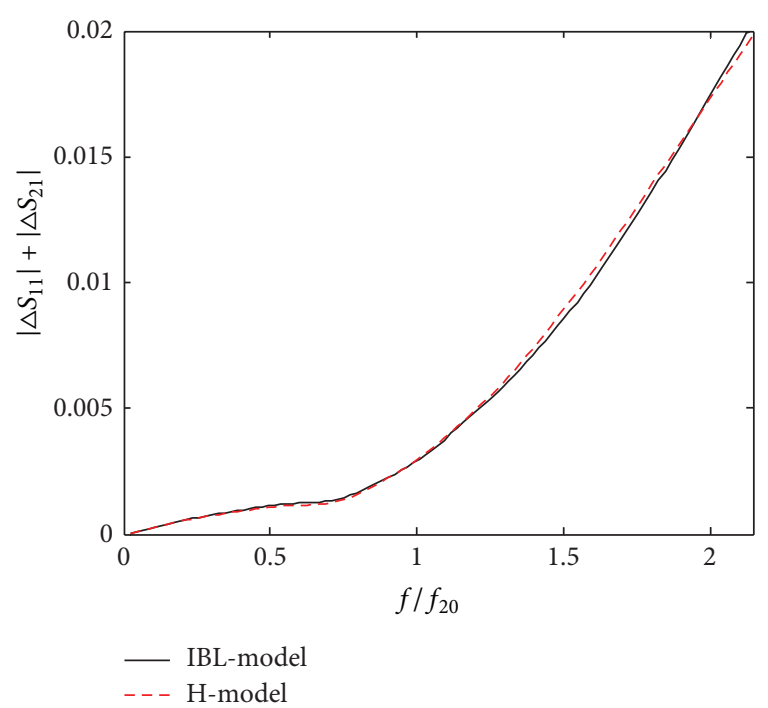

Figure 8: The $S$-parameter differences at $\theta_{0}=60^{\circ}$ for the $\mathrm{H}$ - and IBL-models with parameters retrieved at normal incidence.

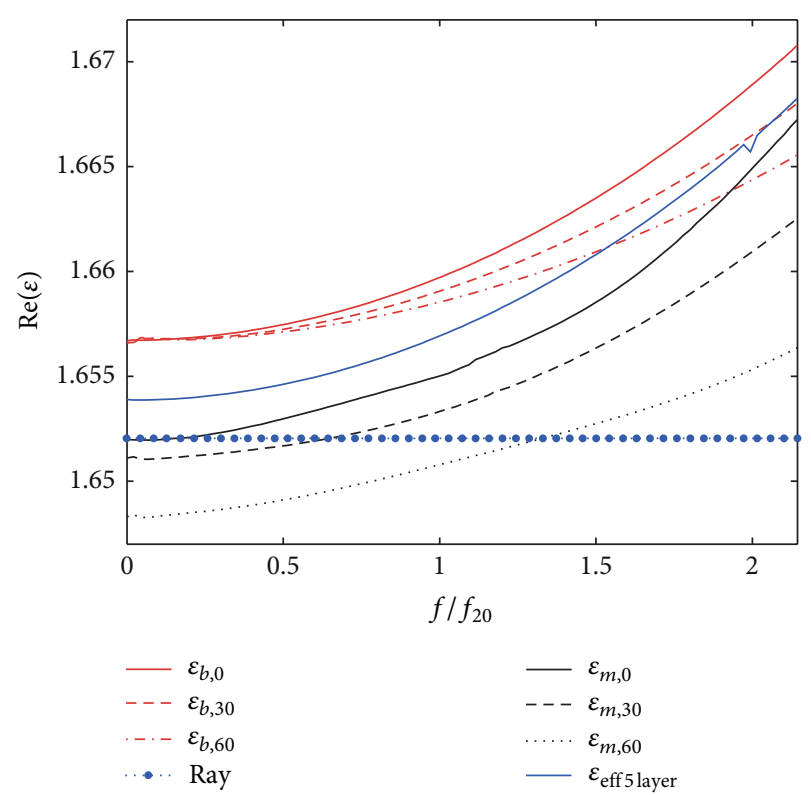

FIGURE 9: Retrieved permittivities of the inner and boundary layers at various incidence angles. The $\varepsilon_{\text {eff } 5 \text { layer }}$ curve denotes the effective permittivity of the $\mathrm{H}$-model for the 5-layer slab; Rayleigh prediction is shown with dot marks; and the subscripts numeral, $b$, and $m$ stand for the incidence angle, boundary layer, and inner layer, respectively.

reasonable. These unexpected phenomena could result from the imprecision introduced by the 2-layer method. However, it is clearly shown that the parameters of the IBL-model are also angle dependent.

In order to determine the angle-dependent parameters $\varepsilon_{b}$ and $\varepsilon_{m}$, we have to first determine $\varepsilon_{b}$ for different $\theta_{0}$ by resolving the angle-dependent $\varepsilon_{\text {eff }}$ of the $\mathrm{H}$-model for a 2-layer slab using (8). Then $\varepsilon_{m}$ can be determined using the retrieved $\varepsilon_{b}$ at each $\theta_{0}$ and (13)-(18). The results are 
supplemented into Figure 9. It is shown that, different from $\varepsilon_{b}$, the retrieved $\varepsilon_{m}$ for different $\theta_{0}$ do not converge to the same value at low frequencies, which is not due to the imprecision of the 2-layer method since any potential homogenization inaccuracies including the retrieval method and spatial dispersion can be neglected in the static or quasistatic region. Hence, this low-frequency divergence in Figure 9 indicates that the stratified model with isotropic boundary layers has inherent deficiencies in describing the dielectriccomposite slabs shown in Figure 1 at oblique incidence.

We have so far considered the performances of two isotropic models (the $\mathrm{H}$ - and IBL-models), which are not sufficient to describe the composite when oblique incidence is of interest. Therefore, the anisotropy will hereafter be taken into account and supplemented into both models.

4.3. Anisotropic Model (A-Model). A straightforward way to determine the parameters of the A-model is to compute the $x$ - and $y$-components of the permittivity $\varepsilon_{x}$ and $\varepsilon_{y}$ by (12). The retrieval results, however, suffer severely from the FPRs and display unphysical behavior [26]. Another way to resolve $\varepsilon_{x}$ and $\varepsilon_{y}$ has to be applied. It is obvious that the A-model will reduce to the $\mathrm{H}$-model at normal incidence, leading to that $\varepsilon_{y}$ equals $\varepsilon_{\text {eff }}\left(\theta_{0}=0^{\circ}\right)$. Assuming that the model parameters are angle independent, $\varepsilon_{y}$ will then be fixed as $\varepsilon_{\text {eff }}\left(\theta_{0}=0^{\circ}\right)$, and $\varepsilon_{x}$ can be calculated using (12) for different $\theta_{0}$. Figure 10 shows that the retrieved $\varepsilon_{x}$ not only decreases with increasing frequency, which violates causality, but also is dependent on $\theta_{0}$, which indicates that the parameters of the A-model are dependent on the angle of incidence. Therefore, this angle dependence has to be complemented to both $\varepsilon_{x}$ and $\varepsilon_{y}$. A closer examination on (6) and (12) reveals that $\varepsilon_{y}$ and $\varepsilon_{\text {eff }}$ are actually identical before the compensation, which makes it a good approximation to let $\varepsilon_{y}\left(\theta_{0}\right)$ equal the compensated angle-dependent $\varepsilon_{\text {eff }}\left(\theta_{0}\right)$ of the H-model. We can then solve $\varepsilon_{x}$ by (12). Figure 11 visualizes the frequency-dependent $\varepsilon_{x}$ and $\varepsilon_{y}$ retrieved at different $\theta_{0}$. At low frequencies, $\varepsilon_{x}$ and $\varepsilon_{y}$ curves converge to two different values, one of which $\left(\varepsilon_{y}\right)$ is larger than the Rayleigh result while the other $\left(\varepsilon_{x}\right)$ is smaller. As the frequency grows, the angle dependence of $\varepsilon_{x}$ and $\varepsilon_{y}$ becomes more and more visible, indicating that the anisotropy cannot effectively describe spatial dispersion.

4.4. Stratified Model with Anisotropic Boundary Layers (ABLModel). We first attempt to determine the parameters of the ABL-model on the basis of the IBL-model, since at normal incidence these two models are identical. If the parameters of the ABL-model are angle-independent, $\varepsilon_{b y}$ of the boundary layer and $\varepsilon_{m}$ of the inner layer should thus equal $\varepsilon_{b}\left(\theta_{0}=0\right)$ and $\varepsilon_{m}\left(\theta_{0}=0\right)$ of the IBL-model, respectively. We can then solve $\varepsilon_{b x}$ for different $\theta_{0}$ using (13)-(18). Similarly to Figure 10, the retrieved $\varepsilon_{b x}$ violates causality and is dependent on the incidence angle. Alternatively, we can fix $\varepsilon_{m}$ of the ABLmodel as $\varepsilon_{m}\left(\theta_{0}=0\right)$ of the IBL-model and then retrieve $\varepsilon_{b x}$ and $\varepsilon_{b y}$ numerically for different $\theta_{0}$ using (13)-(16). The retrieved results shown in Figure 12 are, however, severely influenced by the FPRs. At low frequencies the $\varepsilon_{b y}$ curves converge to the same static value and so do the $\varepsilon_{b x}$ curves

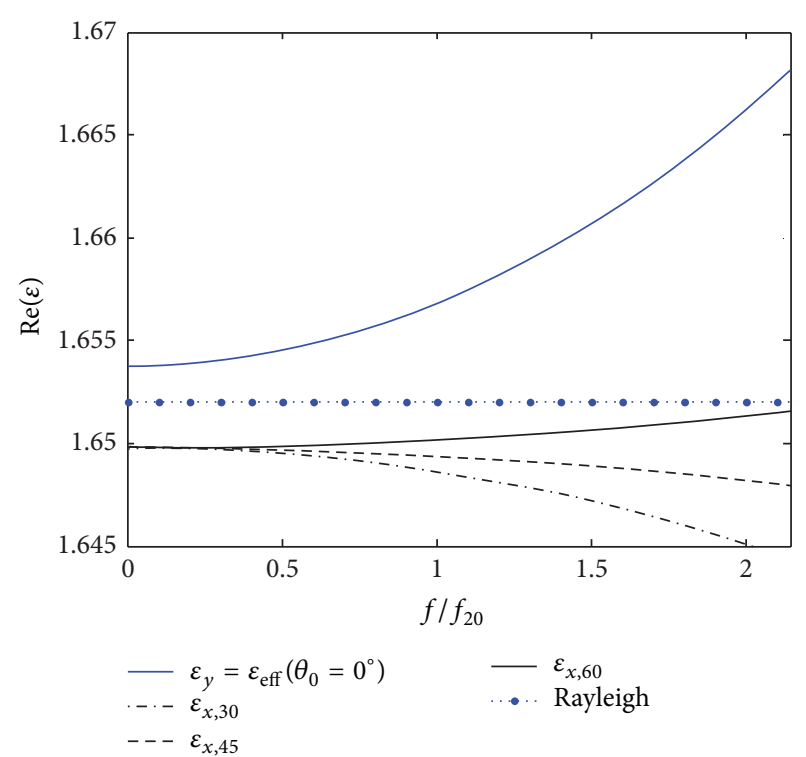

FIGURE 10: Retrieved $\varepsilon_{x}$ of the A-model for various incidence angles when $\varepsilon_{y}$ is fixed as $\varepsilon_{\text {eff }}\left(\theta_{0}=0\right)$. The subscript numerals denote the incidence angles.

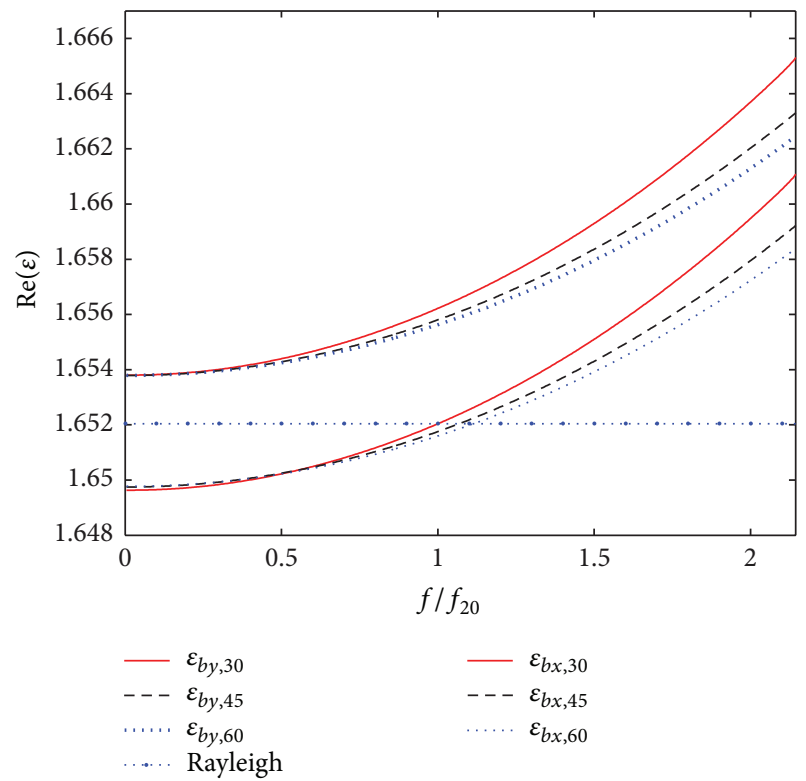

FIGURE 11: Retrieved $\varepsilon_{x}$ and $\varepsilon_{y}$ for different $\theta_{0}$ when $\varepsilon_{y}\left(\theta_{0}\right)$ of the Amodel equals $\varepsilon_{\text {eff }}\left(\theta_{0}\right)$ of the $\mathrm{H}$-model. The subscript numerals denote the incidence angles.

but to a smaller one. As frequency grows, both $\varepsilon_{b y}$ and $\varepsilon_{b x}$ display the angle-dependent character until the appearance of the FPR, which has a broadband influence on the results and thus makes them useless. It is also noted that the FPRs appear at different frequencies for various $\theta_{0}$, since the effective slab thickness $\left(5 a \cdot \cos \theta_{1}\right)$ varies with altering $\theta_{0}$.

As discussed in Section 3.2, we can instead resolve $\varepsilon_{b x}$ and $\varepsilon_{b y}$ for a certain $\theta_{0}$ by computing $\varepsilon_{x}$ and $\varepsilon_{y}$ of the A-model for a 2-layer slab at the same $\theta_{0}$. As for $\varepsilon_{m}$, two scenarios are 


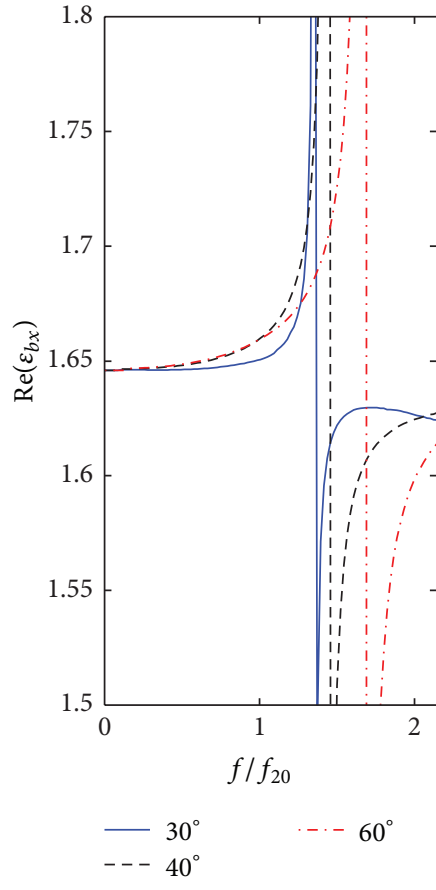

(a)

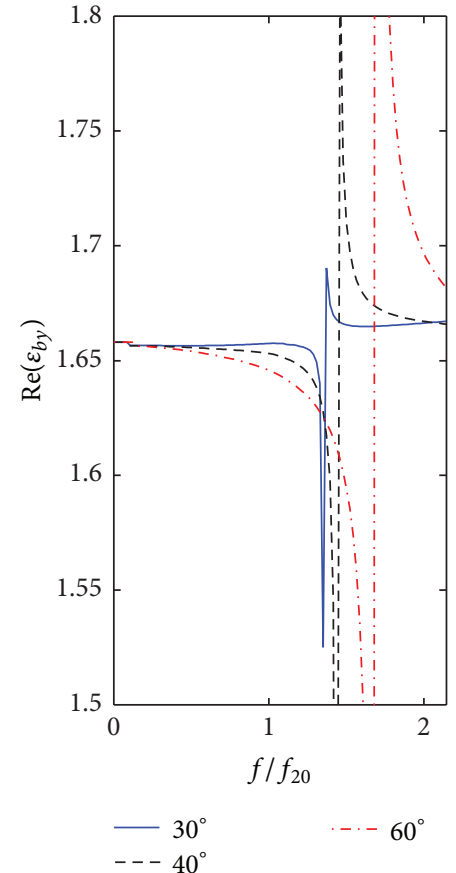

(b)

FIGURE 12: Retrieved $\varepsilon_{b x}$ and $\varepsilon_{b y}$ for various $\theta_{0}$ by (13)-(16) under the condition that $\varepsilon_{m}$ is angle independent and equals $\varepsilon_{m}\left(\theta_{0}=0\right)$ of the IBL model.

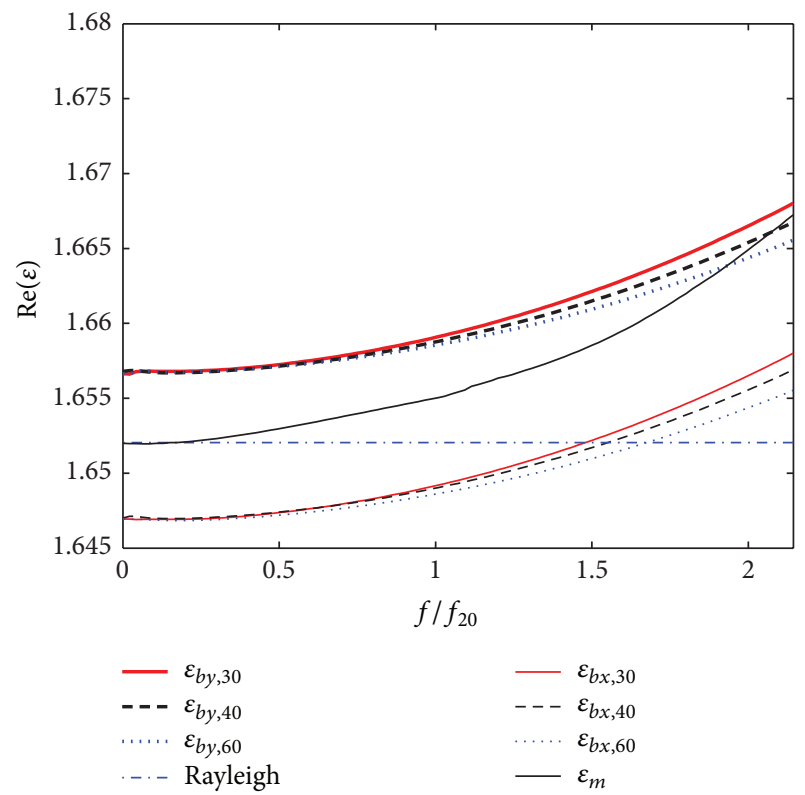

FIGURE 13: Retrieved angle dependent $\varepsilon_{b x}$ and $\varepsilon_{b y}$ of the boundary layer for different $\theta_{0}$. The inner layer $\varepsilon_{m}$ is fixed and equals $\varepsilon_{m}\left(\theta_{0}=0\right)$ of IBL-model. The subscript numerals denote the incidence angles.

considered: one is to keep $\varepsilon_{m}$ angle-independent and equal to $\varepsilon_{m}\left(\theta_{0}=0\right)$ of the IBL-model and the other is to numerically solve $\varepsilon_{m}$ for different $\theta_{0}$ using (13)-(18), given the retrieved $\varepsilon_{b x}$ and $\varepsilon_{b y}$ by the 2-layer method. The acquired permittivities for

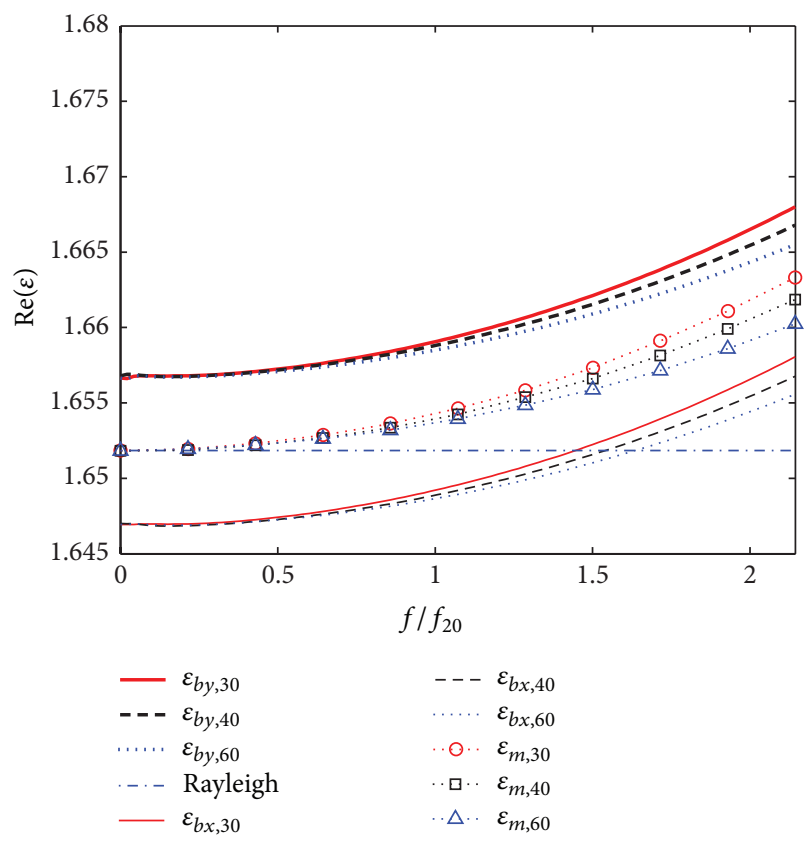

FIGURE 14: Retrieved angle-dependent $\varepsilon_{b x}$ and $\varepsilon_{b y}$ of the boundary layer and $\varepsilon_{m}$ of the inner layer for different incidence angles. The subscript numerals denote the incidence angles.

these two scenarios are then visualized in Figures 13 and 14, respectively. In order to evaluate the above two scenarios of the ABL-model, the $S$-parameter comparisons at $\theta_{0}=30^{\circ}$ and 


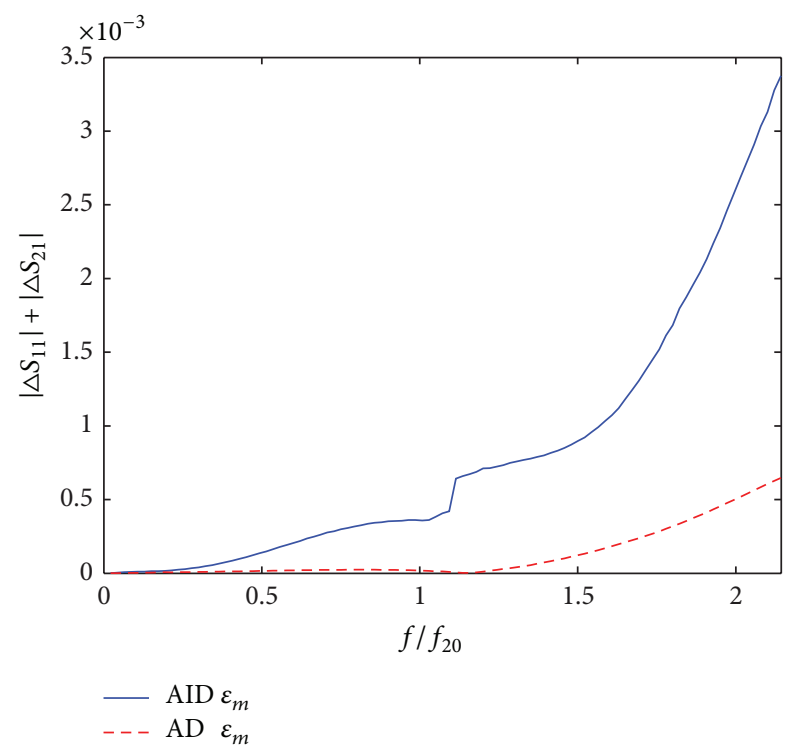

Figure 15: The $S$-parameter differences at $\theta_{0}=30^{\circ}$ for two ABLmodels. Solid curve: the case with angle-independent (AID) $\varepsilon_{m}$ (= $\varepsilon_{m}\left(\theta_{0}=0\right)$ of IBL-model); dashed curve: with angle-dependent (AD) $\varepsilon_{m}$.

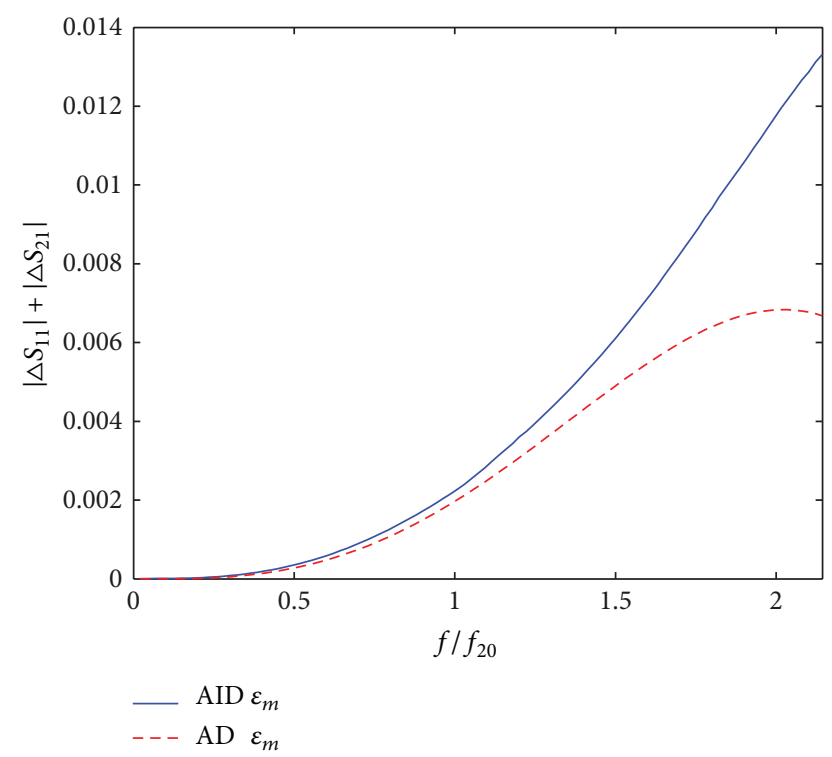

Figure 16: The $S$-parameter differences at $\theta_{0}=60^{\circ}$ for two ABLmodels. Solid curve: the case with angle-independent (AID) $\varepsilon_{m}$ $\left(=\varepsilon_{m}\left(\theta_{0}=0\right)\right.$ of IBL-model); dashed curve: with angle-dependent (AD) $\varepsilon_{m}$.

$60^{\circ}$ are performed and shown in Figures 15 and 16. It is clear that the ABL-model in scenario two beats that in scenario one, which indicates that the angle dependence has to be introduced to the inner layer of the ABL-model in order to achieve better performance.

It is important to notice from Figure 14 that, due to the introduction of the anisotropic boundary layers instead of the isotropic ones, all the retrieved $\varepsilon_{m}$ curves converge

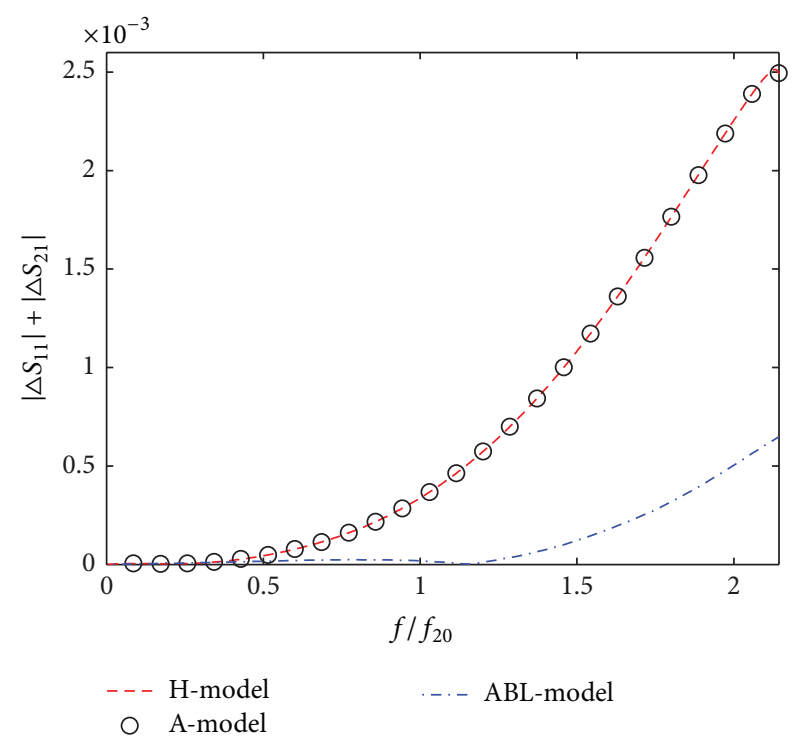

FIgURE 17: The $S$-parameter differences at $\theta_{0}=30^{\circ}$ for three proposed models with angle-dependent parameters. Dashed-red curve: H-model; solid-black curve: A-model; dash-dotted-blue curve: ABL-model.

and gradually approach the static Rayleigh estimate with decreasing frequency. The comparison between the retrieved $\varepsilon_{m}$ for different $\theta_{0}$ in Figures 9 and 14 indicates that if the stratified homogenization model is applied to the dielectriccomposite slab at oblique incidence, the separated boundary layer should be anisotropic in order to ensure that the retrieved model parameters are physically reasonable.

4.5. Necessity of the Additional Boundary Layers and the Anisotropy. It has been so far demonstrated that the parameters of the applied four models are all dependent on the incidence angle $\theta_{0}$. In other words, spatial dispersion makes it very challenging to describe the slab of our interest upon oblique incidence by a model with angle-independent parameters. The additionally introduced anisotropy has not succeeded in characterizing spatial dispersion effectively, and as shown in Figures 11 and 14, the extra $\varepsilon_{x}$ and $\varepsilon_{b x}$ are also dependent on incidence angles. It is also worth mentioning that imaginary parts of retrieved permittivities of all homogenization models are practically zero and therefore not visualized in this paper.

The isotropic $\mathrm{H}_{-}$and IBL-models, with angleindependent parameters, have been compared with each other in Figures 6-8, which reveals the failure of employing the separated isotropic boundary layers. As for the anisotropic models (the $\mathrm{A}$ - and ABL-ones), several attempts to equip each of them with angle-independent parameters have failed. Hence, in order to assess the necessity of introducing the anisotropic boundary layers, the $S$-parameter comparison based on the FPMM can be conducted at different $\theta_{0}$ for three models (the $\mathrm{H}_{-}, \mathrm{A}-$-, and ABL-ones) with derived angle-dependent parameters, as shown in Figures 17 and 18. The IBL-model is not taken 


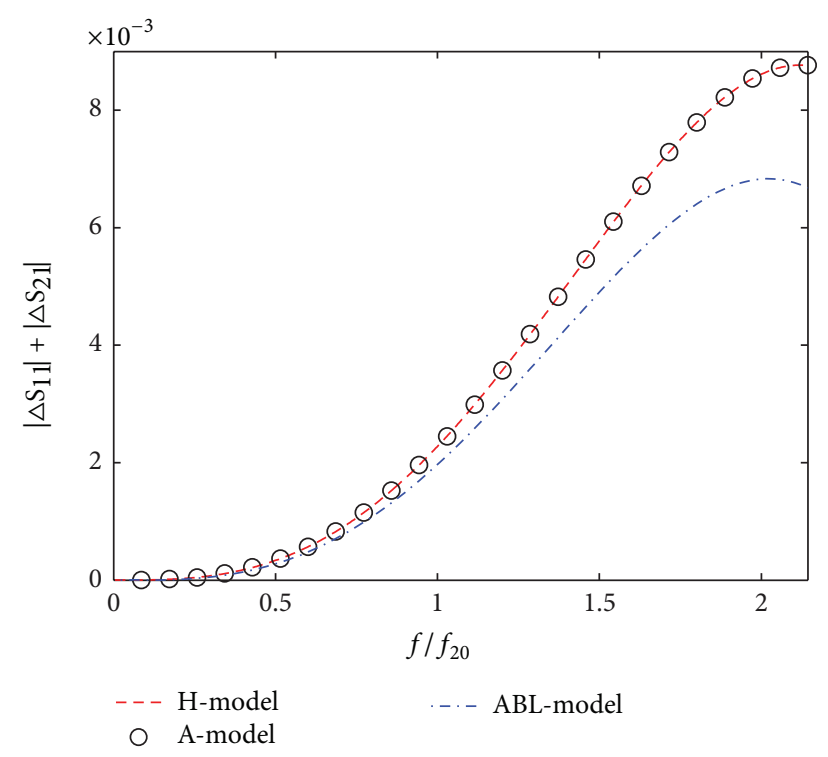

FIgURE 18: The $S$-parameter differences at $\theta_{0}=60^{\circ}$ for three proposed models with angle-dependent parameters. Dashed-red curve: H-model; solid-black curve: A-model; dash-dotted-blue curve: ABL-model.

into account since the retrieved angle-dependent model parameters exhibit unreasonable behaviors, that is, the low frequency divergence of the retrieved $\varepsilon_{m}$, shown in Figure 9.

Several interesting phenomena can be observed in Figures 17 and 18. Firstly, the comparison between the H- and the A-models with angle-dependent parameters shows that the anisotropy alone does not bring in any improvements. Together with Figure 9, it is clear that neither the isotropic boundary layer nor the anisotropy alone is sufficient to improve the model performance. However, the ABL-model clearly beats the $\mathrm{H}$ - and A-models, which confirms that the boundary layers need to be anisotropic if the stratified homogenization model is applied. Secondly, all these three models, despite of angle-dependent parameters, produce larger errors with increasing $\theta_{0}$. This phenomenon could arise from the finite number of the layers composing the slab in the $x$-direction. As $\theta_{0}$ increases, the electrical response in the $x$-direction will gradually dominate. However, only five layers of unit cells exist in this direction. This fact prevents the slab from being homogenized properly. The deterioration of the model performance could therefore be expected for a large $\theta_{0}$. Finally, the compensation method is aimed at restoring physically sound dispersive permittivities for the dielectric slabs. Inevitably, it brings into the system some errors measured by $\left|\Delta S_{11}\right|+\left|\Delta S_{21}\right|$. If the $S$-parameters are in turn calculated from the $\mathrm{H}$ - and the A-models with derived angle-dependent parameters, the errors will be included by $z^{\prime}$. On the other hand, $n^{\prime}$ is not influenced, and moreover two models have the identical $n^{\prime}$. From (6) and (10), it is found that $z^{\prime}=n^{\prime}\left(\varepsilon_{\text {eff }} \cos \theta_{0}\right)^{-1}$ for the H-model and $z^{\prime}=n^{\prime}\left(\varepsilon_{y} \cos \theta_{0}\right)^{-1}$ for the A-model. The generalized impedances of these two models are then identical since $\varepsilon_{y}\left(\theta_{0}\right)=\varepsilon_{\text {eff }}\left(\theta_{0}\right)$. It can therefore be expected that, despite the introduction of an extra parameter, the A-model has the same performance as the H-model when they both have angle-dependent parameters. Nevertheless, the A-model is certainly superior to the $\mathrm{H}$ model with angle-independent parameters retrieved at $\theta_{0}=$ $0^{\circ}$.

\section{Conclusion}

The parameters of four different homogenization models, proposed for a 2D dielectric slab with circular cylindrical inclusions illuminated obliquely by a $\mathrm{TM}_{x y}$-polarized plane wave, have been shown in this paper to be dependent on the incidence angle $\theta_{0}$. With angle-independent parameters retrieved at $\theta_{0}=0^{\circ}$, the isotropic $\mathrm{H}$ - and IBL-models behave similarly for oblique incidence. However, the comparison among three models with angle-dependent parameters has shown that the introduction of neither the separated isotropic boundary nor the anisotropy alone obviously enhances the model quality. More importantly, the boundary layer has to be anisotropic when the stratified model is applied to describe the composite slab. It has also been found that with increasing $\theta_{0}$ all the models generate larger errors in terms of the $S$ parameters, regardless of whether the model parameters are dependent on or independent of the angle of incidence. This phenomenon is due to the finite number of the layers constituting the slab in the $x$-direction.

It should be noticed that all the homogenization processes have been conducted in a quasidynamic range [25], where the homogenization models and the relevant retrieval methods are still meaningful. It had been illustrated in Figures 5, 9, 11, and 14 that, even within this frequency range, the influence of spatial dispersion becomes gradually apparent, which is the fundamental reason that we fail to acquire an angleindependent model. A closer examination of these figures reveals that spatial dispersion is rather negligible when $f / f_{20}$ is very small; for instance, $f / f_{20} \leq 0.5$. Below such a threshold, all these models should have similar performances. More importantly, they are approximately independent of incidence angle. Figures 6-8 and 15-18 also demonstrate these points.

In this paper, the generalized NRW and the compensation methods, when a plane wave excitation at oblique incidence is considered, have been given and shown as useful approaches to retrieve different components of the effective permittivity of dielectric composites, whose geometrical details are fairly small compared with the effective wavelength in the mixtures. Moreover, the 2-layer method was applied for the IBL- and the ABL-models to obtain the boundary layer permittivities. The FPMM also played an important role in calculating the $S$ parameters for a plane wave obliquely incident on the models with the corresponding derived parameters.

\section{Conflict of Interests}

The authors declare that there is no conflict of interests regarding the publication of this paper. 


\section{Acknowledgments}

This work was partially supported by National Natural Science Foundation of China under Grant no. 61301013 and Initialization Research Foundation of Harbin Institute of Technology for employed talented young researchers.

\section{References}

[1] W. S. Weiglhofer and A. Lakhtakia, Introduction to Complex Mediums for Optics and Electromagnetics, vol. PM123, SPIE Press, Bellingham, Wash, USA, 2003.

[2] G. W. Milton, The Theory of Composites, Cambridge Monographs on Applied and Computational Mathematics, Cambridge University Press, Cambridge, UK, 1st edition, 2002.

[3] S. Zouhdi, A. Sihvola, and M. Arsalane, Eds., Advances in Electromagnetics of Complex Media and Metamaterials, vol. 89 of NATO Science Series II: Mathematics, Physics and Chemistry, Springer, Amsterdam, The Netherlands, 2002.

[4] C. Brosseau, "Modelling and simulation of dielectric heterostructures: a physical survey from an historical perspective," Journal of Physics D, vol. 39, no. 7, pp. 1277-1294, 2006.

[5] N. Engheta and R. W. Ziolkowski, Eds., Metamaterials: Physics and Engineering Explorations, IEEE Press, Piscataway, NJ, USA, 2006.

[6] A. Sihvola, Electromagnetic Mixing Formulas and Applications, vol. 47 of IEE Electromagnetic Waves Series, IET, London, UK, 1999.

[7] D. R. Smith and J. B. Pendry, "Homogenization of metamaterials by field averaging," Journal of the Optical Society of America B, vol. 23, no. 3, pp. 391-403, 2006.

[8] A. M. Nicolson and G. F. Ross, "Measurement of the intrinsic properties of materials by time-domain techniques," Transactions on Instrumentation and Measurement, vol. 19, no. 4, pp. 377-382, 1970.

[9] W. B. Weir, "Automatic measurement of complex dielectric constant and permeability at microwave frequencies," Proceedings of the IEEE, vol. 62, no. 1, pp. 33-36, 1974.

[10] D. R. Smith, S. Schultz, P. Markoš, and C. M. Soukoulis, "Determination of effective permittivity and permeability of metamaterials from reflection and transmission coefficients," Physical Review B, vol. 65, no. 19, Article ID 195104, 5 pages, 2002.

[11] X. Chen, T. M. Grzegorczyk, B.-I. Wu, J. Pacheco Jr., and J. A. Kong, "Robust method to retrieve the constitutive effective parameters of metamaterials," Physical Review E, vol. 70, no. 1, Article ID 016608, 7 pages, 2004.

[12] D. R. Smith, D. C. Vier, T. Koschny, and C. M. Soukoulis, "Electromagnetic parameter retrieval from inhomogeneous metamaterials," Physical Review E, vol. 71, no. 3, Article ID 036617, 11 pages, 2005.

[13] C. R. Simovski and S. A. Tretyakov, "Local constitutive parameters of metamaterials from an effective-medium perspective," Physical Review B, vol. 75, no. 19, Article ID 195111, 10 pages, 2007.

[14] C. R. Simovski, "Material parameters of metamaterials," Optics and Spectroscopy, vol. 107, no. 5, pp. 726-753, 2009.

[15] A. I. Cǎbuz, D. Felbacq, and D. Cassagne, "Spatial dispersion in negative-index composite metamaterials," Physical Review A, vol. 77, no. 1, Article ID 013807, 11 pages, 2008.
[16] C. Menzel, C. Rockstuhl, T. Paul, F. Lederer, and T. Pertsch, "Retrieving effective parameters for metamaterials at oblique incidence," Physical Review B, vol. 77, no. 19, Article ID 195328, 8 pages, 2008.

[17] J. Zhou, L. Zhang, G. Tuttle, T. Koschny, and C. M. Soukoulis, "Negative index materials using simple short wire pairs," Physical Review B, vol. 73, no. 4, Article ID 041101, 4 pages, 2006.

[18] T.-C. Yang, Y.-H. Yang, and T.-J. Yen, "An anisotropic negative refractive index medium operated at multiple-angle incidences," Optics Express, vol. 17, no. 26, pp. 24189-24197, 2009.

[19] G. D. Mahan and G. Obermair, "Polaritons at surfaces," Physical Review, vol. 183, no. 3, pp. 834-841, 1969.

[20] C. R. Simovski, S. A. Tretyakov, A. H. Sihvola, and M. M. Popov, "On the surface effect in thin molecular or composite layers," The European Physical Journal Applied Physics, vol. 9, no. 3, pp. 195-204, 2000.

[21] H. Kettunen, J. Qi, H. Wallén, and A. Sihvola, "Homogenization of thin dielectric composite slabs: techniques and limitations," Applied Computational Electromagnetics Society Journal, vol. 26, no. 3, pp. 179-187, 2011.

[22] C. Menzel, T. Paul, C. Rockstuhl, T. Pertsch, S. Tretyakov, and F. Lederer, "Validity of effective material parameters for optical fishnet metamaterials," Physical Review B, vol. 81, no. 3, Article ID 035320, 5 pages, 2010.

[23] J. W. Rayleigh, "On the influence of obstacles arranged in rectangular order upon the properties of a medium," Philosophical Magazine Series, vol. 34, no. 211, pp. 481-502, 1892.

[24] Y. Goykhman and M. Moghaddam, "Retrieval of parameters for three-layer media with non-smooth interfaces for subsurface remote sensing," International Journal of Antennas and Propagation, vol. 2012, Article ID 563730, 12 pages, 2012.

[25] J. Qi, H. Kettunen, H. Wallén, and A. Sihvola, "Quasi-dynamic homogenization of geometrically simple dielectric composites," Applied Computational Electromagnetics Society Journal, vol. 25, no. 12, pp. 1036-1045, 2010.

[26] J. Qi, H. Kettunen, H. Wallén, and A. Sihvola, "Compensation of Fabry-Pérot resonances in homogenization of dielectric composites," IEEE Antennas and Wireless Propagation Letters, vol. 9, pp. 1057-1060, 2010.

[27] J. A. Kong, Electromagnetic Wave Theory, EMW, Cambridge, UK, 2008. 

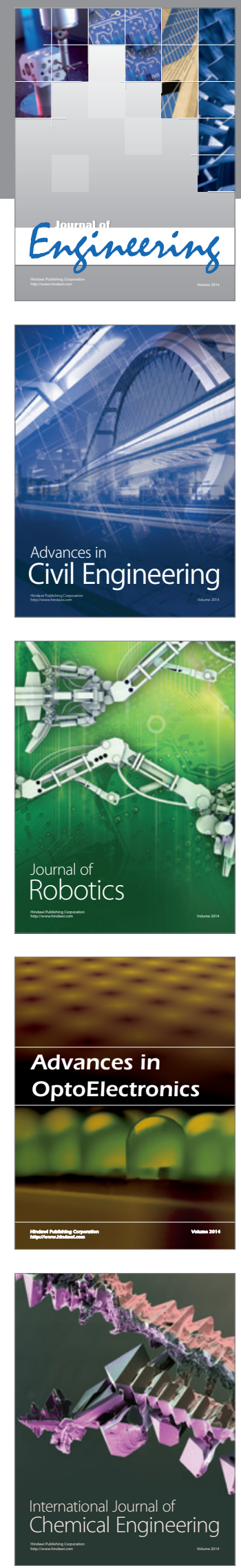

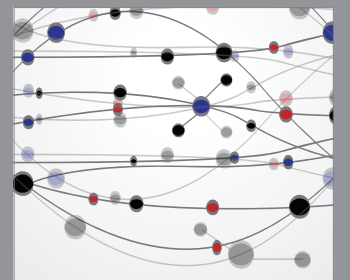

The Scientific World Journal
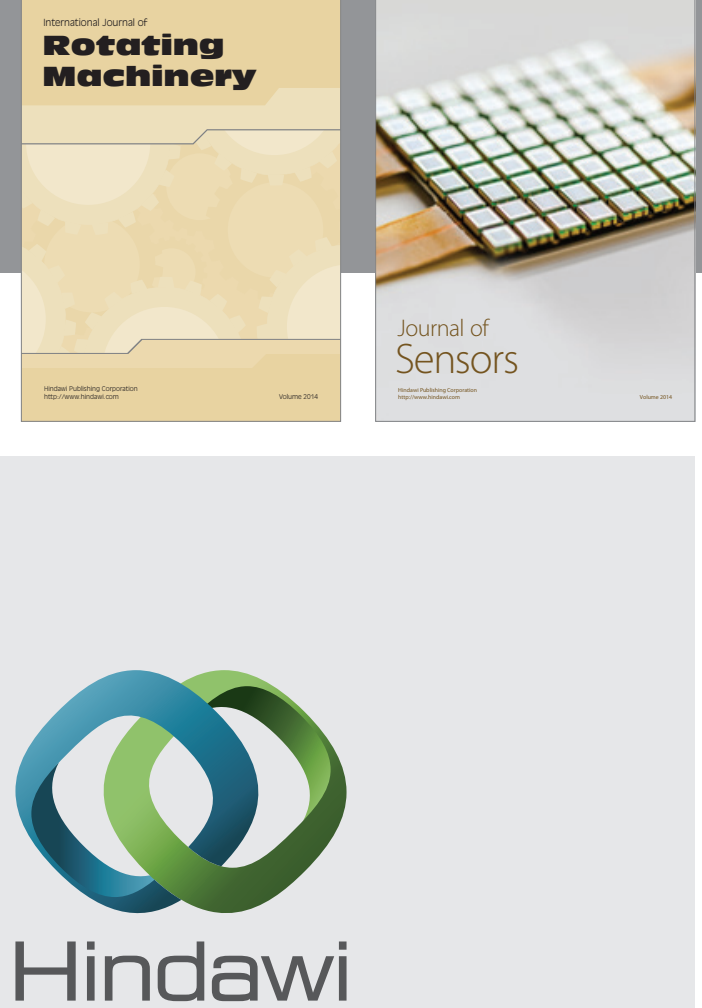

Submit your manuscripts at http://www.hindawi.com
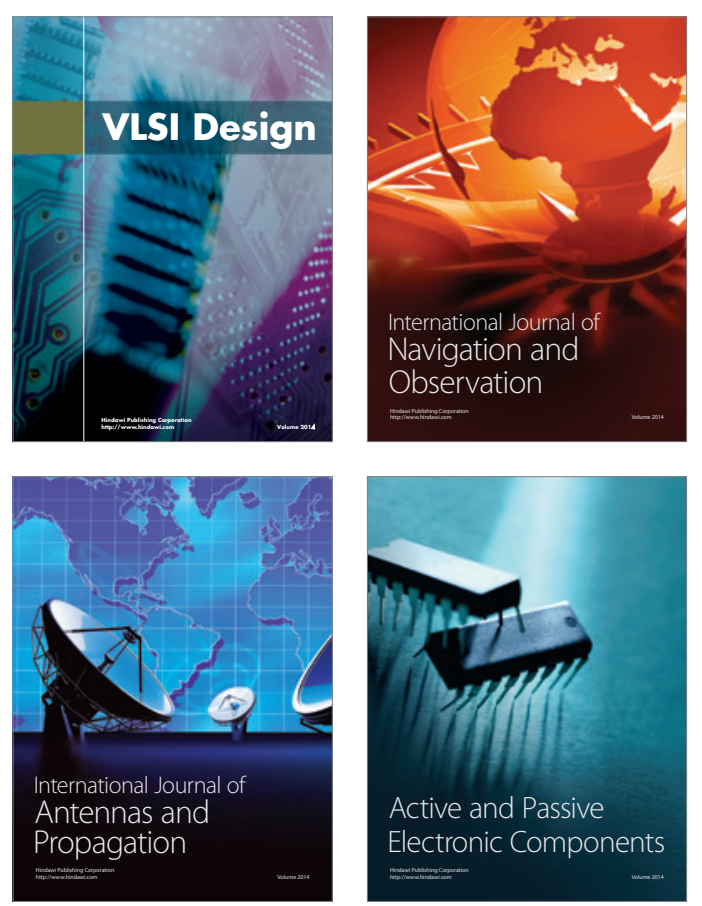
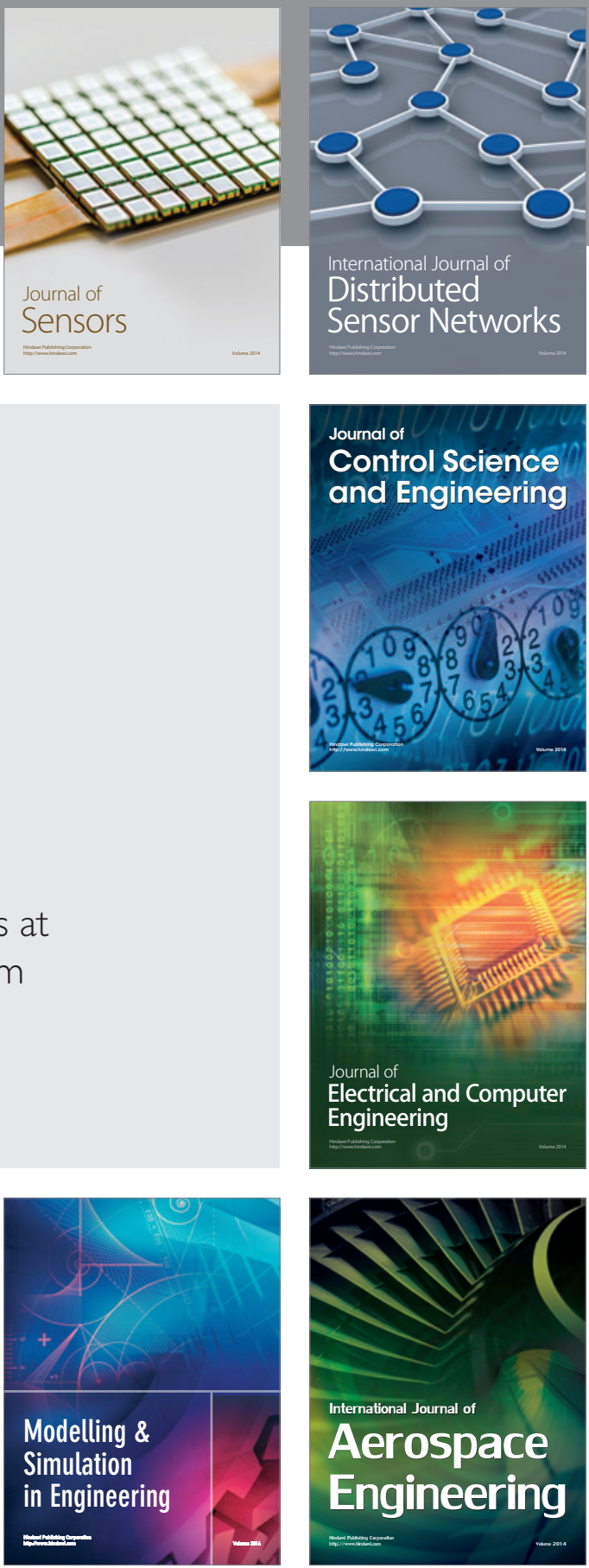

Journal of

Control Science

and Engineering
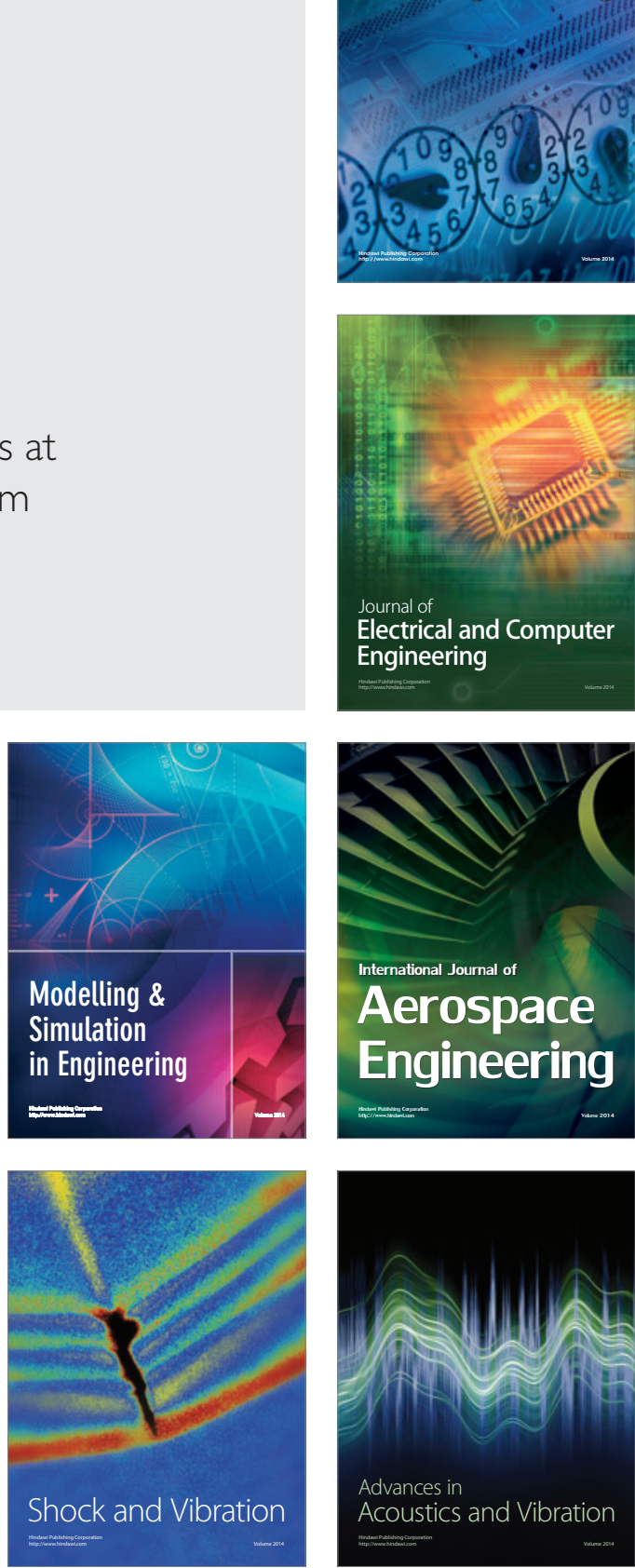